\title{
Difficulties in assessing HIV-prevention interventions in resource-limited settings by randomized controlled trials (RCT) - The example of prevention of mother-to-child transmission (PMTCT)
}

\author{
Isabelle De Vincenzi*, Philippe Gaillard and Tim Farley
}

Address: Department of Reproductive Health and Research, World Health Organization, Geneva, Switzerland

* Corresponding author

from Fourth Dominique Dormont International Conference. Host-Pathogen Interactions in Chronic Infections

Paris, France. 13-15 December 2007

Published: 9 April 2008

Retrovirology 2008, 5(SuppI I):PI3 doi:I0.II86/I742-4690-5-SI-PI3

This abstract is available from: http://www.retrovirology.com/content/5/SI/PI3

(c) 2008 De Vincenzi et al.; licensee BioMed Central Ltd.

Testing new interventions to reduce the MTCT risk in resource-limited settings with an RCT faces various challenges. Pregnant and lactating women with an advanced HIV disease require long-term antiretroviral treatment (ART) for their own health, which reduces substantially MTCT risk. For those not eligible for ART, PMTCT interventions from the $3^{\text {rd }}$ trimester with combination ARVs and modified infant feeding practices greatly reduces risk. At present such MTCT-prevention interventions remain too complex to be considered as standard of care in resource-limited settings. However, in an RCT those interventions must be provided to all participants in the control arm. The residual MTCT risk is therefore low (considerably less than 10\%), and the sample size needed to demonstrate the benefit of any new intervention is now much larger (at least 1000 mother-infant pairs) than when pioneer MTCT-prevention interventions were developed.

HIV prevention research, and MTCT in particular, is a rapidly evolving field. However, conducting an RCT requires time: protocols often have to be adapted to reflect interim findings from other studies, thus delaying the conduct of an RCT and making interpretation of results more complex. When finally available RCT results may be less relevant, as practice evolves, frequently based on results from observational studies.
The cost of such RCTs is high due to: the large sample size needed; the need to ensure HIV care during and after the trial; the large multidisciplinary team needed to ensure care of the participants, analyse specimens and data, and ensure adequate documentation for Good Clinical Practice standards; the need for a multi-site study to achieve the sample size as rapidly as possible, thus increasing staff and infrastructure. The expected benefit/cost ratio of conducting an RCT for testing MTCT-prevention interventions may therefore become ethically questionable. Securing funding for such trials becomes more difficult as most funds are directed at implementing and expanding PMTCT interventions already proven effective and expanding access to HIV care. Therefore, future RCTs on MTCT prevention in resource-limited settings may be hard to implement. The same may apply to other HIV-prevention research. 